\title{
Universiteit
}

Leiden

The Netherlands

\section{Crossover from weak localization to weak antilocalization in a disordered microbridge}

Crawford, M.G.A.; Brouwer, P.W.; Beenakker, C.W.J.

\section{Citation}

Crawford, M. G. A., Brouwer, P. W., \& Beenakker, C. W. J. (2003). Crossover from weak localization to weak antilocalization in a disordered microbridge. Physical Review B, 67(11), 115313. doi:10.1103/PhysRevB.67.115313

Version: $\quad$ Not Applicable (or Unknown)

License: $\quad$ Leiden University Non-exclusive license

Downloaded from: https://hdl.handle.net/1887/76597

Note: To cite this publication please use the final published version (if applicable). 


\title{
Crossover from weak localization to weak antilocalization in a disordered microbridge
}

\author{
M. G. A. Crawford and P. W. Brouwer \\ Laboratory of Atomic and Solid State Physics, Cornell University, Ithaca, New York 14853-2501 \\ C. W. J. Beenakker \\ Instituut-Lorentz, Leiden University, P.O. Box 9506, 2300 RA Leiden, The Netherlands
}

(Received 18 September 2002; published 17 March 2003)

\begin{abstract}
We calculate the weak localization correction in the double crossover to broken time-reversal and spinrotational symmetry for a disordered microbridge or a short disordered wire using a scattering-matrix approach. Whereas the correction has universal limiting values in the three basic symmetry classes, the functional form of the magnetoconductance is affected by eventual nonhomogeneities in the microbridge.

DOI: 10.1103/PhysRevB.67.115313

PACS number(s): 73.20.Fz, 73.21.Hb, 73.23.-b, 73.43.Qt
\end{abstract}

Interference of time-reversed paths causes a small negative quantum correction to the conductance of a disordered metal termed the weak localization. ${ }^{1-4}$ This correction is suppressed by a time-reversal symmetry breaking magnetic field, whereas in the presence of strong spin-orbit scattering, the sign of the correction is reversed..$^{5}$ In that case, the interference correction is known as weak antilocalization.

In a wire geometry at zero temperature, the weak localization correction takes a particularly simple and universal form $^{6}$

$$
\delta G=\frac{2 e^{2}(\beta-2)}{3 \beta h},
$$

where the symmetry parameter $\beta$ denotes the appropriate symmetry class: In the presence of an applied magnetic field $\beta=2$ and without a magnetic field $\beta=4$ or 1 with or without strong spin-orbit scattering, respectively. Equation (1) was obtained using random-matrix theory, ${ }^{7-9}$ and diagrammatic perturbation theory, ${ }^{4,8}$ and is valid if the length $L$ of the wire is much smaller than the localization length $\xi$ and the dephasing length $L_{\phi}$, but much larger than the mean free path $l$. The validity of Eq. (1) extends to the case when sample parameters are nonhomogeneous, e.g., for wires of varying cross section, mean free path, or electron density. ${ }^{10}$

For wires with weak spin-orbit scattering, a crossover between weak localization and weak antilocalization takes place when the spin-orbit scattering length $l_{\text {so }}$ becomes comparable to $L$ or $L_{\phi}$ (whichever is smaller). Experimentally, this crossover regime has been well studied in wires with length $L \gg L_{\phi} \cdot{ }^{11-13}$ In this regime, weak (anti)localization takes the form of a small correction to the conductivity of the wire, rather than of a correction to the conductance. Theoretically, the weak localization to weak antilocalization crossover in the regime $L \gg L_{\phi}$ has been considered in Refs. 14-16 using diagrammatic perturbation theory. The opposite regime $L \ll L_{\phi}$, where the universal correction (1) to the conductance $G$ can be observed, would be relevant for relatively short high-purity metal wires, ${ }^{17}$ or disordered microbridges.

The goal of this paper is threefold: (i) to generalize the random-matrix methods for quantum wires to the crossover between weak localization and weak antilocalization, thus extending the equivalence of the two methods to the interpo- lation between the three symmetry classes, (ii) to find an explicit expression for $\delta G$ for $L \ll L_{\phi}$, and (iii) to extend the theory for the crossover regime to the case of nonhomogeneous wires, for which the electron density, impurity concentration, or cross section varies along the sample. In this case, both the crossover scale and the functional form of $\delta G$ in the crossover are affected by nonhomogeneities. The fact that the crossover scale, characterized by the spin-orbit length $l_{\mathrm{SO}}$ and the magnetic length $l_{H}$, is nonuniversal is well known, both for homogeneous and for nonhomogeneous microbridges. ${ }^{18}$ Our finding that the functional form of the crossover is affected by the nonhomogeneity is markedly different from crossovers between the three basic symmetry classes in quantum dots, where the functional forms are universal and given by random-matrix theory. ${ }^{6}$ For homogeneous wires, $\delta G$ is a universal function of $L / l_{\text {So }}$ and $L / l_{H}$.

The main assumption underlying our calculations is that the wire width $W \ll L$, i.e., quasi-one-dimensionality. We also assume that the wire is well in the diffusive regime, $l$ $\ll L, l_{\text {SO }}, l_{H} \ll \xi$, where $l$ is the elastic mean free path, and, for a nonhomogeneous microbridge, that the number of propagating channels at the Fermi level $N$ has only one minimum along the wire (excluding the possibility of a "cavity"). We first discuss our calculations for homogeneous wires; the case of nonhomogeneous samples is discussed at the end of this paper.

Starting point of our calculation is a random-matrix model similar to that used by Dorokhov. ${ }^{19}$ A disordered wire with $N$ propagating channels at the Fermi level is modeled by $N$ one-dimensional channels and periodically inserted scatterers that scatter within and between the channels. The electronic wavefunction is represented by a $2 N$-component vector of spinors. The $2 N$ components of the wavefunction refer to the transverse channel and to the left/right mover index. Linearizing the kinetic energy in each of the channels, the Hamiltonian $H$ takes the form of a differential operator with respect to the coordinate $x$ along the wire and a $2 N$-dimensional quaternion matrix with respect to the channel and left/right mover indices and spinor degree of freedom

$$
H=-i \sigma_{0} \otimes \tau_{3} \otimes 1_{N} \frac{\partial}{\partial x}+\sum_{j} V_{j} \delta(x-j a),
$$


with $\sigma_{0}$ the $2 \times 2$ unit matrix for the spinor degree of freedom, $\tau_{3}$ the Pauli matrix in left-mover/right-mover grading, $\mathbb{1}_{N}$ the $N \times N$ unit matrix in the channel grading, $V_{j}$ a Hermitian $2 N \times 2 N$ quaternion matrix representing the $j$ th scatterer along the wire, and $a$ the distance between scatterers. A quaternion is a $2 \times 2$ matrix acting in the spinor grading with special rules for transposition and complex conjugation: ${ }^{20}$ The "dual" $X^{R}$ of a quaternion matrix is $X^{R}=\sigma_{2} X^{\mathrm{T}} \sigma_{2}$; the quaternion complex conjugate is defined as $X^{*}=\left(X^{\dagger}\right)^{R}$. We have chosen units such that the Fermi velocity is one. A model similar to Eq. (2) has been used in Ref. 21 to study weak localization in unconventional superconducting wires.

The ensemble-averaged conductance $\langle G\rangle$ of the wire is given by the Landauer formula

$$
\langle G\rangle=\frac{e^{2}}{h} g, \quad g=\left\langle\operatorname{tr}\left(1-r^{\dagger} r\right)\right\rangle,
$$

where $r$ is the $N \times N$ quaternion reflection matrix of the wire. To calculate $r$, we start from a wire of zero length and add slices of length $a$ at the wire's ends. The scattering matrix of the $j$ th scatterer is

$$
S_{j}=\left(\begin{array}{cc}
t_{j} & r_{j}^{\prime} \\
r_{j} & t_{j}^{\prime}
\end{array}\right)=\frac{2 i-V_{j}}{2 i+V_{j}} .
$$

Hence, if a scatterer is added at the lead end of the wire, the new reflection matrix of the wire is calculated according to the composition rule

$$
r \rightarrow r_{j}+t_{j}^{\prime} r\left(1-r_{j}^{\prime} r\right)^{-1} t_{j}
$$

(A similar composition rule, involving both transmission and reflection matrices of the disordered wire, applies if a scatterer is added at the far end of the wire. ${ }^{6}$ )

In left-mover/right-mover grading, the potential $V_{j}$ is parametrized as

$$
V=\left(\begin{array}{ll}
v_{L L} & v_{L R} \\
v_{R L} & v_{R R}
\end{array}\right)
$$

where $v_{L L}, v_{L R}, v_{R L}$, and $v_{R R}$ are $N \times N$ quaternion matrices

$$
\begin{aligned}
v_{L L}\left(\alpha_{f}, \eta_{f}\right)= & v_{R R}^{*}\left(\alpha_{f},-\eta_{f}\right) \\
= & \sqrt{\frac{a}{l_{f} N}}\left[\left(u_{f}^{0}+\eta_{f} x_{f}\right) \otimes \sigma_{0}\right. \\
& \left.+i \alpha_{f} \sum_{\mu=1}^{3} u_{f}^{\mu} \otimes \sigma_{\mu}\right], \\
v_{L R}\left(\alpha_{b}, \eta_{b}\right)= & v_{R L}^{\dagger}\left(\alpha_{b}, \eta_{b}\right) \\
= & \sqrt{\frac{a}{l(N+1)}}\left[\left(u_{b}^{0}+\eta_{b} x_{b}\right) \otimes \sigma_{0}\right. \\
& \left.+i \alpha_{b} \sum_{\mu=1}^{3} u_{b}^{\mu} \otimes \sigma_{\mu}\right] .
\end{aligned}
$$

In Eq. (7), $u_{f}^{0}$ and $x_{f}$ are random Hermitian $N \times N$ matrices, $u_{f}^{\mu}, \mu=1,2,3$, is a random anti-Hermitian matrix, $u_{b}^{0}$ is a random symmetric matrix, and $u_{b}^{\mu}, \mu=1,2,3$ and $x_{b}$ are random antisymmetric matrices. All of these random matrices have independent and Gaussian distributions with zero mean and unit variance. (Variances are specified for the offdiagonal elements; diagonal elements have double variance for symmetric matrices and are zero for antisymmetric matrices.) The parameters $\alpha_{b}$ and $\alpha_{f}$ describe the strength of the breaking of spin-rotational symmetry. The parameters $\eta_{b}$ and $\eta_{f}$ describe the strength of the breaking of time-reversal symmetry. Finally, $l_{f}$ is the elastic mean free path for forward scattering and $l$ is the transport mean free path.

To find the conductance of the wire we calculate the change of $g$ if one scatterer is added to the wire. To this end, we expand the scattering matrix $S_{j}$ of Eq. (4) in powers of $V_{j}$, use the composition rule (5), and calculate the Gaussian average over the potential $V_{j}$. In the limit $a \ll l$ of weak disorder we thus find

$$
-2 N l \frac{\partial}{\partial L} g=g^{2}-h_{0}+3 h_{1} .
$$

We abbreviated

$$
\begin{gathered}
h_{0}=\left\langle\operatorname{tr}\left(1-r^{\dagger} r\right)\left(1-r^{*} r^{R}\right)\right\rangle, \\
h_{1}=\frac{1}{3} \sum_{\mu=1}^{3}\left\langle\operatorname{tr}\left(1-r^{\dagger} r\right) \sigma_{\mu}\left(1-r^{*} r^{R}\right) \sigma_{\mu}\right\rangle,
\end{gathered}
$$

and omitted terms that vanish in the diffusive regime $l$ $\ll L, l_{\text {SO }}, l_{H} \ll N l$. The subscripts 0 and 1 refer to singlet and triplet contributions, respectively.

To leading order in $N$, Eq. (8) can be solved without the interference corrections $h_{0}$ and $h_{1}$, with the result

$$
g=\frac{2 N l}{L}+O(1)
$$

corresponding to the Drude law for the conductance. The $O$ (1) correction in Eq. (10) gives the weak localization correction $\delta g$, which we now compute.

To find the weak localization correction, we need to calculate $h_{0}$ and $h_{1}$. Proceeding as before, we find that the $L$ dependence of $h_{m}, m=0,1$ is governed by the evolution equation

$$
2 N l \frac{\partial h_{m}}{\partial L}=-2\left(\frac{2 N l}{L}+k_{m}\right) h_{m}+\frac{8 N^{2} l^{2}}{L^{2}}, \quad m=0,1
$$

where we abbreviated

$$
k_{0}=\left\langle\operatorname{tr}\left(1-r^{*} r\right)\right\rangle, \quad k_{1}=\frac{1}{3} \sum_{\mu=1}^{3}\left\langle\operatorname{tr}\left(1-r^{*} \sigma_{\mu} r \sigma_{\mu}\right)\right\rangle .
$$

Evolution equations for $k_{0}$ and $k_{1}$ are obtained similarly and read 


$$
\begin{aligned}
& 2 N l \frac{\partial k_{0}}{\partial L}=\left(\frac{2 N l}{l_{H}}\right)^{2}-k_{0}^{2}, \\
& 2 N l \frac{\partial k_{1}}{\partial L}=\left(\frac{2 N l}{l_{H}^{\prime}}\right)^{2}-k_{1}^{2},
\end{aligned}
$$

where the length scales $l_{H}$ and $l_{H}^{\prime}$ are defined in terms of the parameters of the random-matrix model (7),

$$
\begin{gathered}
l_{H}^{-2}=2\left(l^{-2} \eta_{b}^{2}+l^{-1} l_{f}^{-1} \eta_{f}^{2}\right), \\
l_{\mathrm{SO}}^{-2}=6\left(l^{-2} \alpha_{b}^{2}+l^{-1} l_{f}^{-1} \alpha_{f}^{2}\right), \\
\left(l_{H}^{\prime}\right)^{-2}=l_{H}^{-2}+\frac{4}{3} l_{\mathrm{SO}}^{-2} .
\end{gathered}
$$

Equations (11) and (13) have the solution

$$
\begin{gathered}
k_{0}=\frac{2 N l}{l_{H}} \operatorname{cotanh} \frac{L}{l_{H}}, \\
h_{0}=\frac{2 N l}{L}\left(1+\frac{l_{H}}{L} \operatorname{cotanh} \frac{L}{l_{H}}-\operatorname{cotanh}^{2} \frac{L}{l_{H}}\right) .
\end{gathered}
$$

Expressions for $k_{1}$ and $h_{1}$ are obtained from Eq. (15) after the substitution $l_{H} \rightarrow l_{H}^{\prime}$. Substitution of $h_{0}$ and $h_{1}$ into Eq. (8) then allows for the calculation of the weak-localization correction to the conductance

$$
\delta g=\frac{l_{H}}{L} \operatorname{cotanh} \frac{L}{l_{H}}-\frac{l_{H}^{2}}{L^{2}}-3\left(\frac{l_{H}^{\prime}}{L} \operatorname{cotanh} \frac{L}{l_{H}^{\prime}}-\frac{\left(l_{H}^{\prime}\right)^{2}}{L^{2}}\right) .
$$

At zero magnetic field, Eq. (16) simplifies to

$$
\delta g=\frac{1}{3}+\frac{9 l_{\mathrm{SO}}^{2}}{4 L^{2}}-\frac{3 l_{\mathrm{SO}} \sqrt{3}}{2 L} \operatorname{cotanh} \frac{2 L}{l_{\mathrm{SO}} \sqrt{3}} .
$$

Equation (17) reproduces the limits $\delta G=-2 e^{2} / 3 h$ without spin-orbit scattering and $\delta G=e^{2} / 3 h$ with strong spinorbit scattering. Without spin-orbit scattering, Eq. (16) agrees with the weak localization correction calculated in Ref. 22. For large magnetic fields, $L \gg l_{H}$, Eq. (16) simplifies to

$$
\delta g=\frac{1}{L}\left[l_{H}-3\left(l_{H}^{-2}+\frac{4}{3} l_{\mathrm{SO}}^{-2}\right)^{-1 / 2}\right],
$$

which has the same functional form as the weak localization obtained using diagrammatic perturbation theory. ${ }^{14-16,23}$ Comparison of Eq. (18) and Refs. 14-16,23 allows us to identify $l_{\text {SO }}$ as the spin-orbit length, and, for a channel (with width $W \gg l$ ) in a two-dimensional electron gas in a perpendicular magnetic field $B$,

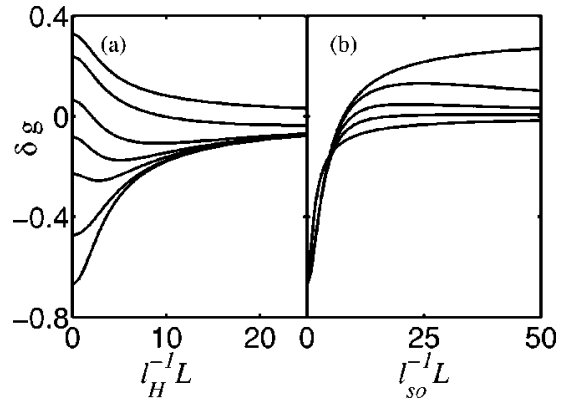

FIG. 1. The weak localization correction $\delta g$ plotted (a) as a function of the magnetic field strength (characterized by the dimensionless ratio $l_{H}^{-1} L$ ) for fixed value of the spin-orbit scattering rate (characterized by $l_{\mathrm{SO}}^{-1} L$ ). From bottom to top, the curves correspond to $L / l_{\mathrm{SO}}=0.1,2,4,6,10,30$, and $\infty$. (b) as a function of length $L$ for fixed $l_{H}^{-1} l_{\mathrm{SO}}$. From bottom to top, the curves correspond to $l_{H}^{-1} l_{\mathrm{SO}}=2,0.3,0.2,0.1$, and 0 .

$$
l_{H}^{2}=3(\hbar / W B e)^{2} .
$$

The case of a cylindrical wire of radius $R \gg l$ and magnetic field perpendicular to the wire is obtained by the substitution $W^{2} \rightarrow 3 R^{2} / 2$. For $l>W$ (or $l>R$ ) the crossover length $l_{H}$ has a more complicated $l$-dependent expression. ${ }^{24}$

Figure 1(a) shows $\delta g$ as a function of the magnetic field for several values of the spin-orbit coupling. In Fig. 1(b) we show $\delta g$ as a function of $l_{\mathrm{SO}}^{-1} L$ for several values of the magnetic field.

We now turn to a description of the weak localization correction in a nonhomogeneous microbridge. Examples of nonhomogeneous microbridges with varying widths are shown in the inset of Fig. 2. If the wire cross section or the electron density vary with the coordinate $x$ along the wire, the number of propagating channels at the Fermi level $N$ also varies with $x$. We assume that $N(x)$ has a minimum for $x$ $=0$ and that $d N / d x>0(d N / d x<0)$ for all $x>0(x<0)$. Further, $x$ dependence of the impurity concentration, the

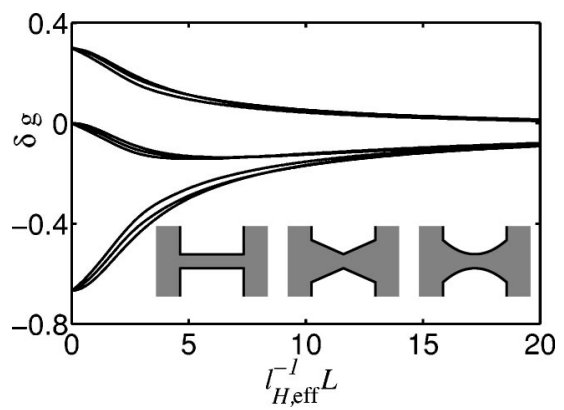

FIG. 2. The weak localization correction $\delta g$ as a function of the magnetic field strength for three different shapes of a disordered microbridge (channels in a two-dimensional electron gas). The three different shapes are characterized by $s(x)=1, s(x)=1+4|2 x / L|$ and $s(x)=1+4(2 x / L)^{2},-L / 2<x<L / 2$, see Eq. (21), as shown in the inset. The three groups of curves correspond to strong, intermediate, and weak spin-orbit scattering from top to bottom, with $l_{\mathrm{SO}}$ in the intermediate case chosen for each case to render the same correction as $l_{H}^{-1} \rightarrow 0$. The magnetic field strength is measured in terms of the effective magnetic length $l_{H \text {, eff }}$, see Eq. (23). 
smoothness of the boundary, the shape of the cross section, etc., causes an $x$ dependence of the length scales $l, l_{H}$, and $l_{\mathrm{SO}}$.

The reflection matrix of the wire is constructed by building the wire from thin slices, starting at the narrowest point $x=0$. This way, the number of channels in the slices added to both ends of the wire can increase, but not decrease. For the construction of an evolution equation for the conductance $g$ and for the auxiliary functions $h_{0}, h_{1}, k_{0}$, and $k_{1}$, we distinguish between two types of added slices: A thin slice that contains a scattering site but for which the number of channels remains constant, and a thin slice without scatterer in which $N$ increases by unity. Addition of a slice of the former type causes a small change in the reflection matrix $r$, which is the same as for a quantum wire of constant thickness, see Eq. (5) above. Addition of a slice for which $N$ increases by unity does not cause a change of the conductance $g$ or of the auxiliary functions $h_{0}, h_{1}, k_{0}$, or $k_{1}$, as can seen by inspecting the cases $x>0$ and $x<0$ separately: For $x>0$, an increase of $N$ does not cause additional reflection, and hence does not affect the reflection matrix $r$; for $x<0$, an increment of $N$ changes the dimension of the reflection matrix $r$ by 1 ,

$$
r \rightarrow\left(\begin{array}{ll}
r & 0 \\
0 & 1
\end{array}\right),
$$

but does not change the conductance $g$ or the functions $h_{0}$, $h_{1}, k_{0}$, or $k_{1}$. Combining the two types of slices, we conclude that the only effect of the $x$ dependence of $N$ and $l$ is indirect, through the explicit appearance of $N$ and $l$ in statistics of the scattering matrix of the added slice, see Eq. (7). In the diffusive regime, $N(x)$ and $l(x)$ only appear in the combination

$$
s(x)=N(x) l(x) / N_{0} l_{0},
$$

where $N_{0}$ and $l_{0}$ are number of propagating channels and mean free path at $x=0$. For large $N$ the function $s(x)$ may be considered continuous, and the evolution equations become differential equations which now include explicit reference to the function $s(x)$. If the wire length $L$ is replaced by the effective length $\bar{L}$,

$$
\bar{L}=\int \frac{d x}{s(x)},
$$

the evolution equations for $g, h_{0}, h_{1}, k_{0}$, and $k_{1}$ keep the same form as for homogeneous wires, provided we make the substitutions $N \rightarrow N_{0}, L \rightarrow \bar{L}, l \rightarrow l_{0}, l_{H} \rightarrow \overline{l_{H}}=l_{H} / s(x)$, and $l_{\mathrm{SO}} \rightarrow l_{\mathrm{SO}}=l_{\mathrm{SO}} / s(x)$.

The functional form of the leading-in- $N$ contribution to the conductance remains unchanged, $G=\left(e^{2} / h\right)\left(2 N_{0} l_{0} / \bar{L}\right)$. Also, for the limiting cases of no spin-orbit scattering and strong spin-orbit scattering, the weak localization correction $\delta G$ is still given by the universal result Eq. (1). ${ }^{10}$ However, because of the $x$ dependence of the length scales $\overline{l_{H}}$ and $\overline{l_{\mathrm{SO}}}$, $\delta g$ acquires an explicit dependence on the shape of the disordered microbridge or the nonhomogeneity of the mean free path or the electron density in the crossover region between the symmetry classes. For a large magnetic field $\left(l_{H}^{-1} L\right.$ $\gg 1$ ), the weak-localization correction can be found in closed form

$$
\begin{gathered}
\delta g=\frac{1}{\bar{L}}\left(l_{H, \mathrm{eff}}-3 l_{H, \mathrm{eff}}^{\prime}\right), \\
l_{H, \mathrm{eff}}=\frac{1}{\bar{L}} \int \frac{l_{H}(x) d x}{s(x)^{2}}, \quad l_{H, \mathrm{eff}}^{\prime}=\frac{1}{\bar{L}} \int \frac{l_{H}^{\prime}(x) d x}{s(x)^{2}} .
\end{gathered}
$$

Equation (23) simplifies to Eq. (18) in the case of $s(x)$ constant. The same result follows if Eq. (18) is interpreted as a quantum interference correction to the one-dimensional resistivity and $l_{H}$ is taken $x$ dependent. For weaker magnetic fields with $l_{H}^{-1} L$ of order unity, a numerical solution of the evolution equations is required.

In Fig. 2, we show results of a numerical solution of $\delta g$ for the examples $s(x)$ constant, $s(x)=1+4|2 x / L|$ and $s(x)=1+4(2 x / L)^{2}, \quad-L / 2<x<L / 2$. These functional forms correspond to diffusive microbridges in a twodimensional electron gas of the form shown in the inset of Fig. 2 with uniform impurity concentration and mean free path $l \ll W$. The three sets of curves in the figure represent strong, intermediate and weak spin-orbit scattering, respectively. For the intermediate case (middle set of curves in Fig. 2 ), three different values of $l_{\mathrm{SO}}$ were chosen so that the weak-localization correction $\delta g=0$ is equal in the three cases for zero magnetic field. The magnetic field is characterized by the ratio $l_{H \text {, eff }}^{-1} L$, see Eq. (23), in order to remove a spurious shape dependence for the large-field asymptotes. While there is no dependence on the form of the function $s(x)$ in the limiting cases of zero and large magnetic fields, we observe that, indeed, $\delta g$ depends on the precise form of the nonhomogeneity for intermediate magnetic field strengths, although, with proper scaling, the difference between the results for the three cases we considered is less than $10 \%$.

In conclusion, we have shown that the scattering matrix approach to quasi-one-dimensional weak localization can be used to obtain a detailed description of the crossover between the different universality classes. We have recovered some results known from diagrammatic perturbation theory, and have discovered one aspect of the problem that has not been noticed previously: The dependence of the functional form of the crossover on nonhomogeneities in the conductor.

We thank V. Ambegaokar, N. W. Ashcroft, and D. Davidovic for discussions. This work was supported by the NSF under Grant Nos. DMR 0086509 and DMR 9988576, by the Packard Foundation, by the Natural Sciences and Engineering Research Council of Canada, and by the Dutch Science Foundation NWO/FOM. 
${ }^{1}$ P. W. Anderson, E. Abrahams, and T. V. Ramakrishnan, Phys. Rev. Lett. 43, 718 (1979).

${ }^{2}$ L. P. Gor'kov, A. I. Larkin, and D. E. Khmel'nitskii, JETP Lett. 30, 228 (1979).

${ }^{3}$ G. Bergmann, Phys. Rep. 107, 1 (1984).

${ }^{4}$ P. A. Lee and T. V. Ramakrishnan, Rev. Mod. Phys. 57, 287 (1985).

${ }^{5}$ S. Hikami, A. I. Larkin, and Y. Nagaoka, Prog. Theor. Phys. 63, 707 (1980).

${ }^{6}$ C. W. J. Beenakker, Rev. Mod. Phys. 69, 731 (1997).

${ }^{7}$ P. A. Mello, Phys. Rev. Lett. 60, 1089 (1988).

${ }^{8}$ P. A. Mello and A. D. Stone, Phys. Rev. B 44, 3559 (1991).

${ }^{9}$ A. M. S. Macêdo and J. T. Chalker, Phys. Rev. B 46, 14985 (1992).

${ }^{10}$ C. W. J. Beenakker and J. A. Melsen, Phys. Rev. B 50, 2450 (1994).

${ }^{11}$ Ç. Kurdak, A. M. Chang, A. Chin, and T. Y. Chang, Phys. Rev. B 46, 6846 (1992).

${ }^{12}$ J. S. Moon, N. O. Birge, and B. Golding, Phys. Rev. B 56, 15124 (1997).

${ }^{13}$ A. B. Gougam, F. Pierre, H. Pothier, D. Esteve, and N. O. Birge, J. Low Temp. Phys. 118, 447 (2000).
${ }^{14}$ B. Altshuler and A. Aronov in Electron-Electron Interactions in Disordered Systems, edited by A. L. Efros and M. Pollak (NorthHolland, Amsterdam, 1985).

${ }^{15}$ P. Santhanam, S. Wind, and E. Prober, in Proceedings of the Seventeenth International Conference on Low Temperature Physics, edited by W. Eckern, A. Schmid, W. Weber, and H. Wühl (Elsevier, New York, 1984), pp. 495-496.

${ }^{16}$ P. Santhanam, S. Wind, and D. E. Prober, Phys. Rev. B 35, 3188 (1987).

${ }^{17}$ F. Pierre, H. Pothier, D. Esteve, and M. H. Devoret, J. Low Temp. Phys. 118, 437 (2000).

${ }^{18}$ C. W. J. Beenakker and H. van Houten, Solid State Phys. 44, 1 (1991).

${ }^{19}$ O. N. Dorokhov, Phys. Rev. B 37, 10526 (1988).

${ }^{20}$ M. L. Mehta, Random Matrices (Academic, New York, 1991).

${ }^{21}$ P. W. Brouwer, A. Furusaki, and C. Mudry, Phys. Rev. B 67, 014530 (2003).

${ }^{22}$ B. L. Altshuler, A. G. Aronov, and A. Y. Zyuzin, Sov. Phys. JETP 59, 415 (1984).

${ }^{23}$ B. L. Al'tshuler and A. G. Aronov, JETP Lett. 33, 499 (1981).

${ }^{24}$ C. W. J. Beenakker and H. van Houten, Phys. Rev. B 38, 3232 (1988). 